\title{
ON THREE-POINT BOUNDARY VALUE PROBLEM WITH A WEIGHTED INTEGRAL CONDITION FOR A CLASS OF SINGULAR PARABOLIC EQUATIONS
}

\author{
ABDELFATAH BOUZIANI
}

Received 10 May 2002

We deal with a three-point boundary value problem for a class of singular parabolic equations with a weighted integral condition in place of one of standard boundary conditions. First we establish an a priori estimate in weighted spaces. Then, we prove the existence, uniqueness, and continuous dependence of a strong solution.

\section{Introduction}

In $Q=\left\{(x, t) \in \mathbb{R}^{2}: 0<x<b, 0<t<T\right\}$, we consider the following problem: given functions $g, v_{0}, M, m, \mu, \mu_{1}$, and $\mu_{2}$, find the function $v=v(x, t)$ as a solution of

$$
\begin{gathered}
\mathscr{L} v=\frac{\partial v}{\partial t}-\frac{\alpha(t)}{x} \frac{\partial}{\partial x}\left(x \frac{\partial v}{\partial x}\right)=f(x, t), \quad(x, t) \in Q, \\
\ell_{0} v=v(x, 0)=v_{0}(x), \quad 0<x<b, \\
\int_{0}^{a} x v(x, t) d x=m(t), \quad 0<a<b, 0<t<T
\end{gathered}
$$

and, on $x=b$, one of the following conditions:

$$
\begin{array}{cl}
v(b, t)=\mu(t), & 0<t<T, \\
\frac{\partial v(b, t)}{\partial x}=\mu_{1}(t), & 0<t<T, \\
\frac{\partial v(b, t)}{\partial x}+\beta v(b, t)=\mu_{2}(t), & 0<t<T, \beta \in \mathbb{R}_{+}^{*},
\end{array}
$$


together with the compatibility conditions

$$
\begin{gathered}
\int_{0}^{a} x v_{0}(x) d x=m(0), \quad v_{0}(b)=\mu(0), \\
{\left[v_{0}^{\prime}(b)=\mu_{1}(0), v_{0}^{\prime}(b)+\beta v_{0}(b)=\mu_{2}(0), \text { resp. }\right]}
\end{gathered}
$$

We describe the complete investigation for problem (1.1) and (1.2). The investigation is similar for problems (1.1), (1.3) and (1.1), (1.4).

We note that problem (1.1) and (1.2) has not been studied previously. It arises from some physical problems. For instance, if $u$ denotes temperature in a heat conduction problem, then $m(t)$ represents the heat moment in the region $0<x<a$ at time $t$. Problems for second-order singular parabolic equations with two-point boundary values were considered in $[1,6,11]$. In [1], it is treated a problem with homogeneous Dirichlet condition and the integral condition $\int_{0}^{b} v(x, t) d x=0$. As for $[6,11]$, are investigated problems which combine Dirichlet condition with the integral condition $\int_{0}^{b} x^{2} v(x, t) d x=m(t)$, and Neumann condition with the integral condition $\int_{0}^{b} x v(x, t) d x=m(t)$, respectively. However, in $[6,11]$, we cannot replace Dirichlet condition by Neumann or Robin conditions and conversely, owing to operators of multiplication constructed to establish a priori estimates for the considered problems. For other equations with integral boundary condition(s), we refer the reader to $[2,3,4,5,8,7,9]$, and the references therein.

In this paper, we prove that problem (1.1) and (1.2) admits a unique strong solution that depends continuously upon the data. The proof is based on an energy inequality and on the density of the range of the operator associated to the abstract formulation of the stated problem.

The paper is divided as follows. Section 2 is devoted to some preliminaries needed in throughout. In Section 3, we prove the uniqueness and continuous dependence of the solution. Then, in Section 4 we establish the existence of the solution.

\section{Preliminaries}

We start by reducing (1.1) and (1.2) to an equivalent problem with homogeneous boundary conditions. For this purpose, we introduce a new unknown function $u$ which represents the deviation of the function $v(x, t)$ from the function

$$
U(x, t)=\frac{2 a-3 x}{2 a-3 b} \mu(t)+\frac{6(x-b)}{\left(2 a^{3}-3 a^{2} b\right)} m(t) .
$$

Therefore, problem (1.1) and (1.2) becomes: find a function $u=u(x, t)$ solution of 


$$
\begin{gathered}
\mathscr{L} u=\frac{\partial u}{\partial t}-\frac{\alpha(t)}{x} \frac{\partial}{\partial x}\left(x \frac{\partial u}{\partial x}\right)=f(x, t), \\
\ell_{0} u=u(x, 0)=u_{0}(x), \\
\int_{0}^{a} x u(x, t) d x=0, \quad u(b, t)=0
\end{gathered}
$$

with

$$
\int_{0}^{a} x u_{0}(x) d x=0, \quad u_{0}(b)=0 .
$$

Assumption 2.1. For all $t \in[0, T]$, we assume

$$
0<c_{0} \leq \alpha(t) \leq c_{1}, \quad\left|\alpha^{\prime}(t)\right| \leq c_{2} .
$$

Assumption 2.2. For all $t \in[0, T]$, we assume

$$
0<c_{3} \leq \alpha^{\prime}(t), \quad\left|\alpha^{\prime \prime}(t)\right| \leq c_{4} .
$$

Throughout the paper, we use the following notation:

$$
\begin{gathered}
Q^{\tau}=(0, b) \times(0, \tau), \quad 0 \leq \tau \leq T ; \\
\mathfrak{J}_{x}^{*} u=\int_{x}^{a} u(\xi, \cdot) d \xi, \quad \mathfrak{J}_{x}^{*^{2}} u=\int_{x}^{a} \int_{\xi}^{a} u(\eta, \cdot) d \eta d \xi, \\
\delta(x)=\left\{\begin{array}{ll}
1, & x \in[0, a], \\
0, & x \in[a, b],
\end{array} \quad \begin{array}{ll}
x^{2}, & x \in[0, a], \\
a^{2}, & x \in[a, b],
\end{array}\right. \\
M u=\frac{w^{\prime}(x)}{x} \mathfrak{I}_{x}^{*}\left(\xi \frac{\partial u}{\partial t}\right)+w(x) \frac{\partial u}{\partial t} .
\end{gathered}
$$

It is easy to observe, for $x \in[0, a]$, that

$$
\begin{gathered}
\mathfrak{J}_{0}^{*}(x u)=\mathfrak{J}_{a}^{*}(x u)=\mathfrak{J}_{a}^{*^{2}}(x u)=0, \\
\int_{0}^{a}\left(\mathfrak{J}_{x}^{*} u\right)^{2} d x \leq 4 \int_{0}^{a} x^{2} u^{2} d x .
\end{gathered}
$$

We introduce function spaces needed in our investigation. We denote by $L_{\sigma}^{2}(0, b)$ the weighted Lebesgue space that consists of all measurable functions $u$ equipped with the finite norm

$$
\|u\|_{L_{\sigma}^{2}(0, b)}:=\left(\int_{0}^{b} \sigma(x)|u(x, \cdot)|^{2} d x\right)^{1 / 2}
$$

By $H_{\sigma}^{1}(0, b)$ we denote the weighted Sobolev space defined as the space of all functions $u \in L_{\sigma}^{2}(0, b)$ such that $\partial u / \partial x$ belongs to $L_{\sigma}^{2}(0, b)$. The corresponding 
norm is

$$
\|u\|_{H_{\sigma}^{1}(0, b)}:=\left(\int_{0}^{b} \sigma(x)\left(|u(x, \cdot)|^{2}+\left|\frac{\partial u(x, \cdot)}{\partial x}\right|^{2}\right) d x\right)^{1 / 2}
$$

If $\sigma(x)=1, L_{\sigma}^{2}(0, b)$ and $H_{\sigma}^{1}(0, b)$ are identified with the standard spaces $L^{2}(0, b)$ and $H^{1}(0, b)$, respectively. By $H^{1}\left(0, b ; x^{2} \delta, x\right)$, we denote the weighted Sobolev space with the finite norm

$$
\|u\|_{H^{1}\left(0, b ; x^{2} \delta, x\right)}:=\left(\int_{0}^{b}\left(x^{2} \delta(x)|u(x, \cdot)|^{2}+x\left|\frac{\partial u(x, \cdot)}{\partial x}\right|^{2}\right) d x\right)^{1 / 2}
$$

We denote by $B_{2, x}^{1, *}(0, a)$ the weighted Bouziani space, first introduced in $[5,6]$, with finite norm

$$
\|u\|_{B_{2, x}^{1, *}(0, a)}:=\left(\int_{0}^{a}\left|\mathfrak{J}_{x}^{*}(\xi u)\right|^{2} d x\right)^{1 / 2}
$$

Moreover, we use $C(0, T ; H)$ and $L^{2}(0, T ; H)$ for the sets of continuous and $L^{2}$-integrable mappings $(0, T) \rightarrow H$, respectively.

We, now, write problem (2.2), (2.3), and (2.4) as an operator equation

$$
L u=\left(f, u_{0}\right)
$$

where $L=(\mathscr{L}, \ell)$ is an unbounded operator, with domain $D(L)$, acting from $B$ to $F$, where $D(L)$ is the set of functions $u$ belonging to $L^{2}\left(0, T ; L_{x}^{2}(0, b)\right)$ for which $\partial u / \partial t,(1 / x)(\partial u / \partial x), \partial^{2} u / \partial x^{2}, \partial^{2} u / \partial t \partial x \in L^{2}\left(0, T ; L_{x}^{2}(0, b)\right)$ and $u$ satisfying conditions (2.4), $B$ is the Banach space obtained by completing the set $D(L)$ with respect to the norm

$$
\|u\|_{B}:=\left(\|u\|_{C\left(0, T ; H_{x w}^{1}(0, b)\right)}^{2}+\left\|\frac{\partial u}{\partial t}\right\|_{L^{2}\left(0, T ; L_{x w}^{2}(0, b)\right)}^{2}\right)^{1 / 2}
$$

and $F$ is the Hilbert space $L^{2}\left(0, T ; L_{x}^{2}(0, b)\right) \times H_{x w}^{1}(0, b)$ consisting of all elements $\left(f, u_{0}\right)$ for which the following norm is finite

$$
\left\|\left(f, u_{0}\right)\right\|_{F}=\left(\|f\|_{L^{2}\left(0, T ; L_{x}^{2}(0, b)\right)}^{2}+\left\|u_{0}\right\|_{H_{x w}^{1}(0, b)}^{2}\right)^{1 / 2} .
$$




\section{Uniqueness and continuous dependence}

First, we establish the following energy inequality.

THEOREM 3.1. Under Assumption 2.1, the following estimate holds for any function $u \in D(L):$

$$
\|u\|_{B} \leq c\|L u\|_{F}
$$

where $c$ is a positive constant independent of $u$.

Proof. Taking the scalar product in $L_{x}^{2}(0, b)$ of $(2.2)$ and $M u$, and integrating the result over $(0, \tau)$, we have

$$
\begin{aligned}
\int_{0}^{\tau}(f(\cdot, t), M \bar{u}(\cdot, t))_{L_{x}^{2}(0, b)} d t \\
=\int_{0}^{\tau}\left\|\frac{\partial u(\cdot, t)}{\partial t}\right\|_{L_{x w}^{2}(0, b)}^{2} d t+\int_{0}^{\tau}\left(\frac{\partial u(\cdot, t)}{\partial t}, w^{\prime} \mathfrak{J}_{x}^{*}\left(\xi \frac{\partial \bar{u}(\cdot, t)}{\partial t}\right)\right)_{L^{2}(0, b)} d t \\
\quad-\int_{0}^{\tau}\left(\alpha(t) \frac{\partial}{\partial x}\left(x \frac{\partial u(\cdot, t)}{\partial x}\right), \frac{\partial \bar{u}(\cdot, t)}{\partial t}\right)_{L_{w}^{2}(0, b)} d t \\
\quad-\int_{0}^{\tau}\left(\alpha(t) \frac{\partial}{\partial x}\left(x \frac{\partial u(\cdot, t)}{\partial x}\right), \frac{w^{\prime}}{x} \mathfrak{J}_{x}^{*}\left(\xi \frac{\partial \bar{u}(\cdot, t)}{\partial t}\right)\right)_{L^{2}(0, b)} d t .
\end{aligned}
$$

Integrating by parts the last three terms on the right-hand side of (3.2), we obtain

$$
\begin{aligned}
\int_{0}^{\tau}\left(\frac{\partial u(\cdot, t)}{\partial t},\right. & \left.w^{\prime} \mathfrak{J}_{x}^{*}\left(\xi \frac{\partial \bar{u}(\cdot, t)}{\partial t}\right)\right)_{L^{2}(0, b)} d t \\
= & 2 \int_{0}^{\tau}\left(x \frac{\partial u(\cdot, t)}{\partial t}, \mathfrak{J}_{x}^{*}\left(\xi \frac{\partial \bar{u}(\cdot, t)}{\partial t}\right)\right)_{L^{2}(0, a)} d t \\
= & -\left.\int_{0}^{\tau}\left|\mathfrak{I}_{x}^{*}\left(\xi \frac{\partial u}{\partial t}\right)\right|^{2}\right|_{0} ^{a} d t=0, \\
-\int_{0}^{\tau}\left(\alpha(t) \frac{\partial}{\partial x}\right. & \left.\left(x \frac{\partial u(\cdot, t)}{\partial x}\right), \frac{\partial \bar{u}(\cdot, t)}{\partial t}\right)_{L_{w}^{2}(0, b)} d t \\
= & -\left.\int_{0}^{\tau} x^{3} \alpha(t) \frac{\partial u}{\partial x} \frac{\partial \bar{u}}{\partial t}\right|_{0} ^{a} d t-\left.\int_{0}^{\tau} x a^{2} \alpha(t) \frac{\partial u}{\partial x} \frac{\partial \bar{u}}{\partial t}\right|_{a} ^{b} d t \\
& +2 \int_{0}^{\tau}\left(\alpha(t) \frac{\partial u(\cdot, t)}{\partial x}, \frac{\partial \bar{u}(\cdot, t)}{\partial t}\right)_{L_{x^{2}}^{2}(0, a)} d t \\
& +\int_{0}^{\tau}\left(\alpha(t) \frac{\partial u(\cdot, t)}{\partial x}, \frac{\partial^{2} \bar{u}(\cdot, t)}{\partial t \partial x}\right)_{L_{x w}^{2}(0, b)} d t \\
= & 2 \int_{0}^{\tau}\left(\alpha(t) \frac{\partial u(\cdot, t)}{\partial x}, \frac{\partial \bar{u}(\cdot, t)}{\partial t}\right)_{L_{x^{2}}^{2}(0, a)} d t+\frac{\alpha(\tau)}{2}\left\|\frac{\partial u(\cdot, \tau)}{\partial x}\right\|_{L_{x w}^{2}(0, b)}^{2} \\
& -\frac{\alpha(0)}{2}\left\|u_{0}^{\prime}\right\|_{L_{x w}^{2}(0, b)}^{2}-\frac{1}{2} \int_{0}^{\tau} \alpha^{\prime}(t)\left\|\frac{\partial u(\cdot, t)}{\partial x}\right\|_{L_{x w}^{2}(0, b)}^{2} d t,
\end{aligned}
$$


522 Singular parabolic equation with integral condition

$$
\begin{aligned}
-\int_{0}^{\tau}(\alpha(t) & \left.\frac{\partial}{\partial x}\left(x \frac{\partial u(\cdot, t)}{\partial x}\right), \frac{w^{\prime}}{x} \mathfrak{J}_{x}^{*}\left(\xi \frac{\partial \bar{u}(\cdot, t)}{\partial t}\right)\right)_{L^{2}(0, b)} d t \\
= & -\left.2 \int_{0}^{\tau} \alpha(t) x \frac{\partial u(\cdot, t)}{\partial x} \mathfrak{J}_{x}^{*}\left(\xi \frac{\partial \bar{u}}{\partial t}\right)\right|_{0} ^{a} d t \\
& -2 \int_{0}^{\tau}\left(\alpha(t) \frac{\partial u(\cdot, t)}{\partial x}, \frac{\partial \bar{u}(\cdot, t)}{\partial t}\right)_{L_{x^{2}}^{2}(0, a)} d t
\end{aligned}
$$

Substituting (3.3) into (3.2), yields

$$
\begin{aligned}
2 \int_{0}^{\tau}\left\|\frac{\partial u(\cdot, t)}{\partial t}\right\|_{L_{x w}^{2}(0, b)}^{2} d t+\alpha(\tau)\left\|\frac{\partial u(\cdot, \tau)}{\partial x}\right\|_{L_{x w}^{2}(0, b)}^{2} \\
=2 \int_{0}^{\tau}(f(\cdot, t), M \bar{u}(\cdot, t))_{L_{x}^{2}(0, b)} d t \\
+\alpha(0)\left\|u_{0}^{\prime}\right\|_{L_{x w}^{2}(0, b)}^{2}+\int_{0}^{\tau} \alpha^{\prime}(t)\left\|\frac{\partial u(\cdot, t)}{\partial x}\right\|_{L_{x w}^{2}(0, b)}^{2} d t
\end{aligned}
$$

According to Cauchy inequality and (2.10), the first term on the right-hand side of (3.4) is estimated as follows:

$$
\begin{aligned}
2 \int_{0}^{\tau}( & f(\cdot, t), M \bar{u}(\cdot, t))_{L_{x}^{2}(0, b)} d t \\
\leq & 2 \varepsilon_{1} \int_{0}^{\tau}\|f(\cdot, t)\|_{L_{x}^{2}(0, a)}^{2} d t+\varepsilon_{2} \int_{0}^{\tau}\|f(\cdot, t)\|_{L_{x w}^{2}(0, b)}^{2} d t \\
& +\frac{2}{\varepsilon_{1}} \int_{0}^{\tau}\left\|\frac{\partial u(\cdot, t)}{\partial t}\right\|_{B_{2, x}^{1, *}(0, a)}^{2} d t+\frac{1}{\varepsilon_{2}} \int_{0}^{\tau}\left\|\frac{\partial u(\cdot, t)}{\partial t}\right\|_{L_{x w}^{2}(0, b)}^{2} d t \\
\leq & \left(2 \varepsilon_{1} a+\varepsilon_{2} a^{2}\right) \int_{0}^{\tau}\|f(\cdot, t)\|_{L_{x}^{2}(0, b)}^{2} d t+\left(\frac{8 a}{\varepsilon_{1}}+\frac{1}{\varepsilon_{2}}\right) \int_{0}^{\tau}\left\|\frac{\partial u(\cdot, t)}{\partial t}\right\|_{L_{x w}^{2}(0, b)}^{2} d t
\end{aligned}
$$

Inserting (3.5) into (3.4), and choosing $\varepsilon_{1}$ and $\varepsilon_{2}$ so that the last integral in the right-hand side of (3.5) will be absorbed in the left-hand side of (3.4), we get by virtue of Assumption 2.1 that

$$
\begin{aligned}
& \frac{3}{2} \int_{0}^{\tau}\left\|\frac{\partial u(\cdot, t)}{\partial t}\right\|_{L_{x w}^{2}(0, b)}^{2} d t+c_{0}\left\|\frac{\partial u(\cdot, \tau)}{\partial x}\right\|_{L_{x w}^{2}(0, b)}^{2} \\
& \quad \leq 68 a^{2} \int_{0}^{\tau}\|f(\cdot, t)\|_{L_{x}^{2}(0, b)}^{2} d t+c_{1}\left\|u_{0}^{\prime}\right\|_{L_{x w}^{2}(0, b)}^{2}+c_{2} \int_{0}^{\tau}\left\|\frac{\partial u(\cdot, t)}{\partial x}\right\|_{L_{x w}^{2}(0, b)}^{2} d t .
\end{aligned}
$$


Adding the obvious inequality

$$
\|u(\cdot, \tau)\|_{L_{x w}^{2}(0, b)}^{2} \leq\left\|u_{0}\right\|_{L_{x w}^{2}(0, b)}^{2}+\int_{0}^{\tau}\left(\|u(\cdot, t)\|_{L_{x w}^{2}(0, b)}^{2}+\left\|\frac{\partial u(\cdot, t)}{\partial t}\right\|_{L_{x w}^{2}(0, b)}^{2}\right) d t
$$

to (3.6), we conclude that

$$
\begin{aligned}
& \int_{0}^{\tau}\left\|\frac{\partial u(\cdot, t)}{\partial t}\right\|_{L_{x w}^{2}(0, b)}^{2} d t+\|u(\cdot, \tau)\|_{H_{x w}^{1}(0, b)}^{2} \\
& \quad \leq c_{5}\left(\int_{0}^{\tau}\|f(\cdot, t)\|_{L_{x}^{2}(0, b)}^{2} d t+\left\|u_{0}\right\|_{H_{x w}^{1}(0, b)}^{2}\right)+c_{6} \int_{0}^{\tau}\|u(\cdot, t)\|_{H_{x w}^{1}(0, b)}^{2} d t
\end{aligned}
$$

where

$$
c_{5}=\frac{\max \left(68 a^{2}, c_{1}, 1\right)}{\min \left(1 / 2, c_{0}\right)}, \quad c_{6}=\frac{\max \left(c_{2}, 1\right)}{\min \left(1 / 2, c_{0}\right)} .
$$

Hence, application of Gronwall's type inequality [3, Lemma 1] gives

$$
\begin{aligned}
& \int_{0}^{\tau}\left\|\frac{\partial u(\cdot, t)}{\partial t}\right\|_{L_{x w}^{2}(0, b)}^{2} d t+\|u(\cdot, \tau)\|_{H_{x w}^{1}(0, b)}^{2} \\
& \quad \leq c_{5} \exp \left(c_{6} T\right)\left(\|f\|_{L^{2}\left(0, T ; L_{x}^{2}(0, b)\right)}^{2} d t+\left\|u_{0}\right\|_{H_{x w}^{1}(0, b)}^{2}\right)
\end{aligned}
$$

As the right-hand side of (3.10) is independent of $\tau$, we take the upper bound of the left-hand side. Hence, we obtain the required estimate.

Since we have no information concerning $L B$ except that $L B \subset F$, we extend $L$, so that inequality (3.1) holds for the extension and $\bar{L} B$ is the whole space. For this purpose, we state the following proposition.

Proposition 3.2. Under the hypotheses of Theorem 3.1, the operator $L: B \rightarrow F$ possesses a closure.

Proof. The proof is similar to that in [8].

Let $\bar{L}$ be the closure of the operator $L$, and $D(\bar{L})$ its domain.

Definition 3.3. The solution of equation

$$
\bar{L} u=\left(f, u_{0}\right)
$$

is called strong solution of problem (2.2), (2.3), and (2.4). 
524 Singular parabolic equation with integral condition

Passing to the limit in inequality (3.1), we obtain

$$
\|u\|_{B} \leq c\|\bar{L} u\|_{F}
$$

from which, we have the following corollaries.

Corollary 3.4. Under the hypotheses of Theorem 3.1. If, there exists a strong solution of problem (2.2), (2.3), and (2.4), then it is unique and depends continuously upon $\left(f, u_{0}\right)$.

Corollary 3.5. The range $\bar{L} B$ is closed in $F$, and $\bar{L} B=\overline{L B}$.

\section{Existence of the solution}

Theorem 4.1. Under Assumptions 2.1 and 2.2, problem (2.2), (2.3), and (2.4) admits a unique solution in the sense of Definition 3.3, for arbitrary $f \in L^{2}(0, T$; $\left.L_{x}^{2}(0, b)\right)$ and $u_{0} \in H_{x w}^{1}(0, b)$, such that $u \in C\left(0, T ; H_{x w}^{1}(0, b)\right)$ and $\partial u / \partial t \in L^{2}(0, T$; $\left.L_{x w}^{2}(0, b)\right)$.

Proof. Section 3 implies that $\bar{L}$ is injective. Therefore, to show the existence of the solution, it suffices to prove that $\bar{L}$ is surjective. This can be fulfilled if we establish the density of $L B$ in $F$. To this end, we state the following result which we need below.

Proposition 4.2. Under the hypotheses of Theorem 4.1, if

$$
(\mathscr{L} u, \bar{\omega})_{L^{2}\left(0, T ; L_{x}^{2}(0, b)\right)}=0
$$

for arbitrary $u \in D_{0}(L)=\{u / u \in D(L): \ell u=0\}$ and some $\omega \in L^{2}\left(0, T ; L_{x}^{2}(0, b)\right)$, then $\omega$ vanishes almost everywhere in $Q$.

Suppose that for the moment that Proposition 4.2 has been proved, and turning to the proof of Theorem 4.1. Let the element $\left(f, u_{0}\right) \in F$ be orthogonal to $L B$, that is, let

$$
\left(L u, \overline{\left(f, u_{0}\right)}\right)_{F}=(\mathscr{L u}, \bar{f})_{L^{2}\left(0, T ; L_{x}^{2}(0, b)\right)}+\left(\ell u, \bar{u}_{0}\right)_{H_{x w}^{1}(0, b)}=0, \quad u \in D(L) .
$$

Assuming in (4.2) that $u$ is replaced by any element of $D_{0}(L)$. It follows from Proposition 4.2 that $f=0$. Hence

$$
\left(\ell u, \bar{u}_{0}\right)_{H_{x w}^{1}(0, b)}=0, \quad u \in D(L)
$$

But the set $\ell B$ is everywhere dense in $H_{x w}^{1}(0, b)$. The above relation implies that $u_{0}=0$. Consequently $\overline{L B}=F$. To complete the proof of Theorem 4.1, it remains to prove Proposition 4.2. 
Proof of Proposition 4.2. We start by constructing the function $\omega$. Since equality (4.1) holds for arbitrary element of $D_{0}(L)$, we express it in the following form:

$$
v= \begin{cases}0, & 0 \leq t \leq s \\ \int_{s}^{t} \frac{\partial u}{\partial \tau} d \tau, & s \leq t \leq T,\end{cases}
$$

and $\partial v / \partial t$ is solution of the equation

$$
-\alpha(t) \delta(x) \mathfrak{J}_{x}^{*^{2}}\left(\xi \frac{\partial v}{\partial t}\right)+\alpha(t) \frac{\partial v}{\partial t}=\int_{t}^{T} \omega(x, \tau) d \tau=g,
$$

where $s$ is an arbitrary fixed number in $[0, T]$.

It is easy to see from (4.4) that $v \in D_{s}(L)=\{u / u \in D(L): u=0$ for $t \leq s\} \subseteq$ $D_{0}(L)$, and from (4.5) that $\partial v /\left.\partial t\right|_{t=T}=0$. Differentiating (4.5) with respect to $t$, it yields

$$
\omega(x, t)=\frac{\partial}{\partial t}\left(\alpha(t) \delta(x) \mathfrak{J}_{x}^{*^{2}}\left(\xi \frac{\partial v}{\partial t}\right)-\alpha(t) \frac{\partial v}{\partial t}\right)
$$

Lemma 4.3. Under the hypotheses of Theorem 4.1, the function w defined by (4.6) belongs to $L^{2}\left(s, T ; L_{x}^{2}(0, b)\right)$.

Proof of Lemma 4.3. Set $y(x, t):=\delta(x) \mathfrak{J}_{x}^{*^{2}}(\xi(\partial v / \partial t))-\partial v / \partial t$, then $\omega(x, t)=$ $\alpha^{\prime}(t) y+\alpha(t)(\partial y / \partial t)$. We first prove that $\alpha^{\prime}(t) y$ is bounded in $L^{2}\left(s, T ; L_{x}^{2}(0, b)\right)$. Indeed, we have according to Assumption 2.1

$$
\begin{aligned}
\left\|\alpha^{\prime} y\right\|_{L^{2}\left(s, T ; L_{x}^{2}(0, b)\right)}^{2} & \leq c_{2}^{2}\|y\|_{L^{2}\left(s, T ; L_{x}^{2}(0, b)\right)}^{2} \\
& \leq c_{2}^{2} \int_{s}^{T} \int_{0}^{b} x\left(\delta(x) \mathfrak{J}_{x}^{*^{2}}\left(\xi \frac{\partial v}{\partial t}\right)-\frac{\partial v}{\partial t}\right)^{2} d x d t \\
& \leq 2 c_{2}^{2}\left(\int_{s}^{T} \int_{0}^{a} x\left|\mathfrak{J}_{x}^{*^{2}}\left(\xi \frac{\partial v}{\partial t}\right)\right|^{2} d x d t+\int_{s}^{T} \int_{0}^{b} x(t)\left|\frac{\partial v}{\partial t}\right|^{2} d x d t\right) .
\end{aligned}
$$

By virtue of inequality (2.10), it yields

$$
\begin{aligned}
\left\|\alpha^{\prime} y\right\|_{L^{2}\left(s, T ; L_{x}^{2}(0, b)\right)}^{2} & \leq 32 c_{2}^{2} a^{6}\left\|\frac{\partial v}{\partial t}\right\|_{L^{2}\left(s, T ; L_{x}^{2}(0, a)\right)}^{2}+2 c_{2}^{2}\left\|\frac{\partial v}{\partial t}\right\|_{L^{2}\left(s, T ; L_{x}^{2}(0, b)\right)}^{2} \\
& \leq 2 c_{2}^{2} \max \left(16 a^{6}, 1\right)\left\|\frac{\partial v}{\partial t}\right\|_{L^{2}\left(s, T ; L_{x}^{2}(0, b)\right)}^{2}
\end{aligned}
$$

It remains to prove that $\alpha(t)(\partial y / \partial t)$ belongs to $L^{2}\left(s, T ; L_{x}^{2}(0, b)\right)$. To this end, we must use the following lemma, in which we summarize some of the properties of the averaging operator $\varrho_{\varepsilon}$ defined by

$$
\left(\varrho_{\varepsilon} y\right)(\cdot, t):=\frac{1}{\varepsilon} \int_{s}^{T} \varphi\left(\frac{t-t^{\prime}}{\varepsilon}\right) y\left(\cdot, t^{\prime}\right) d t^{\prime},
$$


526 Singular parabolic equation with integral condition

where $\varphi \in C^{\infty}(\mathbb{R}), \varphi=0$ in the neighborhood of $t=s$ and $t=T$ and outside the interval $(s, T)$, and such that

$$
\left(\frac{1}{\varepsilon}\right) \int_{\mathbb{R}} \varphi\left(\frac{\left(t-t^{\prime}\right)}{\varepsilon}\right) d t^{\prime}=\left(\frac{1}{\varepsilon}\right) \int_{\mathbb{R}} \varphi\left(\frac{\left(t-t^{\prime}\right)}{\varepsilon}\right) d t=1
$$

Lemma 4.4. (i) If $y \in L^{2}(s, T ; H)$, the function $\varrho_{\varepsilon} y \in C^{\infty}(s, T ; H)$;

(ii) if $\left\|\varrho_{\varepsilon} y\right\|_{L^{2}(s, T ; H)} \leq\|y\|_{L^{2}(s, T ; H)}$, and $\left\|\varrho_{\varepsilon} y-y\right\|_{L^{2}(s, T ; H)} \rightarrow 0$ as $\varepsilon \rightarrow 0$;

(iii) $(d / d t) \varrho_{\varepsilon} y=\varrho_{\varepsilon}(d / d t) y, u \in D_{s}(L)$;

(iv) if $y \in L^{2}(s, T ; H)$, then $\left\|(\partial / \partial t)\left(\alpha \varrho_{\varepsilon} y-\varrho_{\varepsilon} \alpha y\right)\right\|_{L^{2}(s, T ; H)} \rightarrow 0$ as $\varepsilon \rightarrow 0$.

Proof. Proof of this lemma is similar to that of [10, Lemma 9.1].

We apply operators $\varrho_{\varepsilon}$ and $\partial / \partial t$ to (4.5), it follows

$$
\alpha(t) \frac{\partial}{\partial t} \varrho_{\varepsilon} y=\frac{\partial}{\partial t}\left(\varrho_{\varepsilon} g\right)+\frac{\partial}{\partial t}\left(\alpha(t) \varrho_{\varepsilon} y-\varrho_{\varepsilon} \alpha(t) y\right)-\alpha^{\prime}(t) \varrho_{\varepsilon} y,
$$

from which, we obtain, using (4.8), and properties (ii), (iii) of Lemma 4.4, that

$$
\begin{gathered}
\left\|\alpha(t) \frac{\partial}{\partial t} \varrho_{\varepsilon} y\right\|_{L^{2}\left(s, T ; L_{x}^{2}(0, b)\right)}^{2} \\
\leq 3\left(\left\|\frac{\partial}{\partial t}\left(\varrho_{\varepsilon} g\right)\right\|_{L^{2}\left(s, T ; L_{x}^{2}(0, b)\right)}^{2}+\left\|\alpha^{\prime} \varrho_{\varepsilon} y\right\|_{L^{2}\left(s, T ; L_{x}^{2}(0, b)\right)}^{2}\right) \\
+\left\|\frac{\partial}{\partial t}\left(\alpha \varrho_{\varepsilon} y-\varrho_{\varepsilon} \alpha y\right)\right\|_{L^{2}\left(s, T ; L_{x}^{2}(0, b)\right)}^{2}+3\left\|\frac{\partial}{\partial t}\left(\alpha \varrho_{\varepsilon} y-\varrho_{\varepsilon} \alpha y\right)\right\|_{L^{2}\left(s, T ; L_{x}^{2}(0, b)\right)}^{2} \\
\leq 3\left\|\frac{\partial g}{\partial t}\right\|_{L^{2}\left(s, T ; L_{x}^{2}(0, b)\right)}^{2} \\
+6 c_{2}^{2} \max \left(16 a^{6}, 1\right)\left\|\frac{\partial v}{\partial t}\right\|_{L^{2}\left(s, T ; L_{x}^{2}(0, b)\right)}^{2} .
\end{gathered}
$$

Since the norm of $\alpha(t)(\partial / \partial t) \varrho_{\varepsilon} y$ is bounded in $L^{2}\left(s, T ; L_{x}^{2}(0, b)\right)$, then we pass to the limit in the above inequality, by taking into account property (iv) in Lemma 4.4, we conclude that

$$
\begin{aligned}
\| \alpha \frac{\partial}{\partial t} & y \|_{L^{2}\left(s, T ; L_{x}^{2}(0, b)\right)}^{2} \\
& \leq \max \left(3,96 c_{2}^{2} a^{6}, 6 c_{2}^{2}\right)\left(\left\|\frac{\partial g}{\partial t}\right\|_{L^{2}\left(s, T ; L_{x}^{2}(0, b)\right)}^{2}+\left\|\frac{\partial v}{\partial t}\right\|_{L^{2}\left(s, T ; L_{x}^{2}(0, b)\right)}^{2}\right) .
\end{aligned}
$$

It then follows from $(4.8)$ and (4.13) that $\omega \in L^{2}\left(s, T ; L_{x}^{2}(0, b)\right)$. 
Substituting (2.2) and (4.6) into (4.1), we get

$$
\begin{aligned}
\left(x \frac{\partial v}{\partial t}\right. & \left., \frac{\partial}{\partial t}\left(\alpha \mathfrak{J}_{x}^{*^{2}}\left(\xi \frac{\partial \bar{v}}{\partial t}\right)\right)\right)_{L^{2}\left(s, T ; L^{2}(0, a)\right)}-\left(x \frac{\partial v}{\partial t}, \frac{\partial}{\partial t}\left(\alpha \frac{\partial \bar{v}}{\partial t}\right)\right)_{L^{2}\left(s, T ; L^{2}(0, b)\right)} \\
& -\left(\alpha \frac{\partial}{\partial x}\left(x \frac{\partial v}{\partial x}\right), \frac{\partial}{\partial t}\left(\alpha \mathfrak{J}_{x}^{*^{2}}\left(\xi \frac{\partial \bar{v}}{\partial t}\right)\right)\right)_{L^{2}\left(s, T ; L^{2}(0, a)\right)} \\
& +\left(\alpha \frac{\partial}{\partial x}\left(x \frac{\partial v}{\partial x}\right), \frac{\partial}{\partial t}\left(\alpha \frac{\partial \bar{v}}{\partial t}\right)\right)_{L^{2}\left(s, T ; L^{2}(0, b)\right)}=0 .
\end{aligned}
$$

Integrating by parts each term of (4.14), by taking into account (4.4) and (4.5), we obtain

$$
\begin{aligned}
& \left(x \frac{\partial v}{\partial t}, \frac{\partial}{\partial t}\left(\alpha \mathfrak{J}_{x}^{*^{2}}\left(\xi \frac{\partial \bar{v}}{\partial t}\right)\right)\right)_{L^{2}\left(s, T ; L^{2}(0, a)\right)} \\
& =\frac{1}{2} \int_{0}^{a} \alpha(s)\left|\mathfrak{I}_{x}^{*}\left(\xi \frac{\partial v(\xi, s)}{\partial t}\right)\right|^{2} d x-\frac{1}{2} \int_{s}^{T} \int_{0}^{a} \alpha^{\prime}(t)\left|\mathfrak{J}_{x}^{*}\left(\xi \frac{\partial v}{\partial t}\right)\right|^{2} d x, \\
& -\left(x \frac{\partial v}{\partial t}, \frac{\partial}{\partial t}\left(\alpha \frac{\partial \bar{v}}{\partial t}\right)\right)_{L^{2}\left(s, T ; L^{2}(0, b)\right)} \\
& =\frac{1}{2} \int_{0}^{b} x \alpha(s)\left|\frac{\partial v(x, s)}{\partial t}\right|^{2} d x-\frac{1}{2} \int_{s}^{T} \int_{0}^{b} x \alpha^{\prime}(t)\left|\frac{\partial v}{\partial t}\right|^{2} d x d t, \\
& -\left(\alpha \frac{\partial}{\partial x}\left(x \frac{\partial v}{\partial x}\right), \frac{\partial}{\partial t}\left(\alpha \mathfrak{J}_{x}^{*^{2}}\left(\xi \frac{\partial \bar{v}}{\partial t}\right)\right)\right)_{L^{2}\left(s, T ; L^{2}(0, a)\right)} \\
& =\int_{s}^{T} \int_{0}^{a} x^{2} \alpha^{2}(t)\left|\frac{\partial v}{\partial t}\right|^{2} d x d t+\frac{1}{2} \int_{0}^{a} x^{2} \alpha(T) \alpha^{\prime}(T)|v(x, T)|^{2} d x \\
& -\frac{1}{2} \int_{s}^{T} \int_{0}^{a} x^{2}\left(\alpha^{\prime 2}(t)+\alpha(t) \alpha^{\prime \prime}(t)\right)|v|^{2} d x d t \\
& -\int_{s}^{T} \int_{0}^{a}\left(\alpha(t) \mathfrak{I}_{x}^{*} \frac{\partial v}{\partial t}+\alpha^{\prime}(t) \mathfrak{I}_{x}^{*} v\right) x \frac{\partial \bar{v}}{\partial t} d x d t \\
& -\left(\alpha \frac{\partial}{\partial x}\left(x \frac{\partial v}{\partial x}\right), \frac{\partial}{\partial t}\left(\alpha \frac{\partial \bar{v}}{\partial t}\right)\right)_{L^{2}\left(s, T ; L^{2}(0, b)\right)} \\
& =\int_{s}^{T} \int_{0}^{b} \alpha^{2}(t) x\left|\frac{\partial^{2} v}{\partial x \partial t}\right|^{2} d x d t+\frac{1}{2} \int_{0}^{b} x \alpha(T) \alpha^{\prime}(T)\left|\frac{\partial v(x, T)}{\partial x}\right|^{2} d x \\
& -\frac{1}{2} \int_{s}^{T} \int_{0}^{b}\left(\alpha^{\prime 2}(t)+\alpha(t) \alpha^{\prime \prime}(t)\right) x\left|\frac{\partial v}{\partial x}\right|^{2} d x d t .
\end{aligned}
$$

It then follows from (4.15) that

$$
2 \int_{s}^{T} \int_{0}^{a} x^{2} \alpha^{2}(t)\left|\frac{\partial v}{\partial t}\right|^{2} d x d t+2 \int_{s}^{T} \int_{0}^{b} x \alpha^{2}(t)\left|\frac{\partial^{2} v}{\partial x \partial t}\right|^{2} d x d t
$$


528 Singular parabolic equation with integral condition

$$
\begin{aligned}
& +\int_{0}^{a} \alpha(s)\left|\mathfrak{J}_{x}^{*}\left(\xi \frac{\partial v(\xi, s)}{\partial t}\right)\right|^{2} d x+\int_{0}^{b} x \alpha(s)\left|\frac{\partial v(x, s)}{\partial t}\right|^{2} d x \\
& +\int_{0}^{a} x^{2} \alpha(T) \alpha^{\prime}(T)|v(x, T)|^{2} d x+\int_{0}^{b} x \alpha(T) \alpha^{\prime}(T)\left|\frac{\partial v(x, T)}{\partial x}\right|^{2} d x \\
& =\int_{s}^{T} \int_{0}^{a} \alpha^{\prime}(t)\left|\mathfrak{I}_{x}^{*}\left(\xi \frac{\partial v}{\partial t}\right)\right|^{2} d x+\int_{s}^{T} \int_{0}^{b} x \alpha^{\prime}(t)\left|\frac{\partial v}{\partial t}\right|^{2} d x d t \\
& +\int_{s}^{T} \int_{0}^{a} x^{2}\left(\alpha^{\prime 2}(t)+\alpha(t) \alpha^{\prime \prime}(t)\right)|v|^{2} d x d t \\
& +\int_{s}^{T} \int_{0}^{b}\left(\alpha^{\prime 2}(t)+\alpha(t) \alpha^{\prime \prime}(t)\right) x\left|\frac{\partial v}{\partial x}\right|^{2} d x d t \\
& -2 \int_{s}^{T} \int_{0}^{a}\left(\alpha(t) \mathfrak{J}_{x}^{*} \frac{\partial v}{\partial t}+\alpha^{\prime}(t) \mathfrak{I}_{x}^{*} v\right) x \frac{\partial \bar{v}}{\partial t} d x d t .
\end{aligned}
$$

Applying the Cauchy inequality and inequality (2.10) to the last term on the right-hand side of (4.16), we obtain

$$
\begin{aligned}
-2 \int_{s}^{T} & \int_{0}^{a}\left(\alpha(t) \mathfrak{I}_{x}^{*} \frac{\partial v}{\partial t}+\alpha^{\prime}(t) \mathfrak{I}_{x}^{*} v\right) x \frac{\partial \bar{v}}{\partial t} d x d t \\
\leq & \int_{s}^{T} \int_{0}^{a} x^{2}\left|\frac{\partial v}{\partial t}\right|^{2} d x d t+2 \int_{s}^{T} \alpha^{2} d t \int_{0}^{a}\left|\mathfrak{I}_{x}^{*} \frac{\partial v}{\partial t}\right|^{2} d x \\
& +2 \int_{s}^{T} \alpha^{\prime 2} d t \int_{0}^{a}\left|\mathfrak{J}_{x}^{*} v\right|^{2} d x \\
\leq & a \int_{s}^{T} \int_{0}^{a} x\left|\frac{\partial v}{\partial t}\right|^{2} d x d t+8 a \int_{s}^{T} \alpha^{2} d t \int_{0}^{a} x\left|\frac{\partial v}{\partial t}\right|^{2} d x \\
& +8 \int_{s}^{T} \alpha^{\prime 2} d t \int_{0}^{a} x^{2}|v|^{2} d x \\
\leq & \int_{s}^{T}\left(a+8 a \alpha^{2}\right) d t \int_{0}^{b} x\left|\frac{\partial v}{\partial t}\right|^{2} d x \\
& +8 \int_{s}^{T} \alpha^{\prime 2} d t \int_{0}^{b}\left(x^{2} \delta(x)|v|^{2}+x\left|\frac{\partial v}{\partial x}\right|^{2}\right) d x
\end{aligned}
$$

Inserting (4.17) into (4.16) and employing Assumptions 2.1 and 2.2, we conclude that

$$
\begin{gathered}
\left\|\frac{\partial v}{\partial t}\right\|_{L^{2}\left(s, T ; H^{1}\left(0, b ; x^{2} \delta, x\right)\right)}^{2}+\left\|\frac{\partial v(\cdot, s)}{\partial t}\right\|_{B_{2, x}^{1, *}(0, a)}^{2}+\left\|\frac{\partial v(\cdot, s)}{\partial t}\right\|_{L_{x}^{2}(0, b)}^{2}+\|v(\cdot, T)\|_{H^{1}\left(0, b ; x^{2} \delta, x\right)}^{2} \\
\quad \leq c_{7} \int_{s}^{T}\left(\left\|\frac{\partial v(\cdot, t)}{\partial t}\right\|_{B_{2, x}^{1, *}(0, a)}^{2}+\left\|\frac{\partial v(\cdot, t)}{\partial t}\right\|_{L_{x}^{2}(0, b)}^{2}+\|v(\cdot, t)\|_{H^{1}\left(0, b ; x^{2} \delta, x\right)}^{2}\right) d t,
\end{gathered}
$$


where

$$
c_{7}=\frac{\max \left(c_{1}, c_{1}+8 a c_{1}^{2}+a, c_{2}^{2}+c_{1} c_{4}+8 c_{1}^{2}\right)}{\min \left(2 c_{0}^{2}, c_{0}, c_{0} c_{3}\right)} .
$$

To continue, we introduce a new function $\theta$ defined by

$$
\theta(x, t):=\int_{t}^{T} \frac{\partial v}{\partial \tau} d \tau
$$

from which we deduce that $\theta(x, s)=v(x, T)$ and $v(x, t)=\theta(x, s)-\theta(x, t)$. Hence, inequality (4.18) becomes

$$
\begin{aligned}
& \left\|\frac{\partial v}{\partial t}\right\|_{L^{2}\left(0, T ; H^{1}\left(0, b, x^{2} \delta, x\right)\right)}^{2}+\left\|\frac{\partial v(\cdot, s)}{\partial t}\right\|_{B_{2, x}^{1, *}(0, a) \cap L_{x}^{2}(0, b)}^{2}+\left(1-2 c_{7}(T-s)\right)\|\theta(\cdot, s)\|_{H^{1}\left(0, b ; x^{2} \delta, x\right)}^{2} \\
& \quad \leq 2 c_{7} \int_{s}^{T}\left(\left\|\frac{\partial v(\cdot, t)}{\partial t}\right\|_{B_{2, x}^{1, *}(0, a) \cap L_{x}^{2}(0, b)}^{2}+\|\theta(\cdot, t)\|_{H^{1}\left(0, b ; x^{2} \delta, x\right)}^{2}\right) d t .
\end{aligned}
$$

If $s_{0}$ verifies $1-2 c_{7}\left(T-s_{0}\right)=1 / 2$, then the above inequality implies

$$
\begin{gathered}
\left\|\frac{\partial v}{\partial t}\right\|_{L^{2}\left(0, T ; H^{1}\left(0, b, x^{2} \delta, x\right)\right)}^{2}+\left\|\frac{\partial v(\cdot, s)}{\partial t}\right\|_{B_{2, x}^{1, *}(0, a) \cap L_{x}^{2}(0, b)}^{2}+\|\theta(\cdot, s)\|_{H^{1}\left(0, b ; x^{2} \delta, x\right)}^{2} \\
\quad \leq 4 c_{7} \int_{s}^{T}\left(\left\|\frac{\partial v(\cdot, t)}{\partial t}\right\|_{B_{2, x}^{1, *}(0, a) \cap L_{x}^{2}(0, b)}^{2}+\|\theta(\cdot, t)\|_{H^{1}\left(0, b ; x^{2} \delta, x\right)}^{2}\right) d t
\end{gathered}
$$

for all $s \in\left[s_{0}, T\right]$. By virtue of [3, Lemma 1] for the interval $(s, T),(4.22)$ implies that

$$
\left\|\frac{\partial v}{\partial t}\right\|_{L^{2}\left(0, T ; H^{1}\left(0, b, x^{2} \delta, x\right)\right)}^{2}+\|\theta(\cdot, s)\|_{H^{1}\left(0, b ; x^{2} \delta, x\right)}^{2}+\left\|\frac{\partial v(\cdot, s)}{\partial t}\right\|_{B_{2, x}^{1, *}(0, a) \cap L_{x}^{2}(0, b)}^{2} \leq 0,
$$

from which we conclude that $\partial v / \partial t \equiv 0$ and thus $\omega \equiv 0$ almost everywhere in $\left(s_{0}, T\right) \times(0, b)$. As $s$ is an arbitrary fixed point in $[0, T]$, using the same reasoning again, from which, step by step, we deduce that $\omega \equiv 0$ almost everywhere in $Q$. This achieves the proof of Theorem 4.1.

\section{References}

[1] N.-E. Benouar and N. I. Yurchuk, A mixed problem with an integral condition for parabolic equations with a Bessel operator, Differentsial'nye Uravneniya 27 (1991), no. 12, 2094-2098 (Russian), translated in Differential Equations 27 (1991), no. $12,1482-1487$.

[2] A. Bouziani, Mixed problem with boundary integral conditions for a certain parabolic equation, J. Appl. Math. Stochastic Anal. 9 (1996), no. 3, 323-330. 
530 Singular parabolic equation with integral condition

[3] On a class of parabolic equations with a nonlocal boundary condition, Acad. Roy. Belg. Bull. Cl. Sci. (6) 10 (1999), no. 1-6, 61-77.

[4] Strong solution to an hyperbolic evolution problem with nonlocal boundary conditions, Maghreb Math. Rev. 9 (2000), no. 1-2, 71-84.

[5] - On the solvability of nonlocal pluriparabolic problems, Electron. J. Differential Equations 2001 (2001), no. 21, 1-16.

[6] - On the solvability of a class of singular parabolic equations with nonlocal boundary conditions in nonclassical function spaces, Int. J. Math. Math. Sci. 30 (2002), no. 7, 435-447.

[7] On a class of composite equations with nonlocal boundary conditions, to appear in Engineering Simulations.

[8] On a quasistatic flexure of a thermoelastic rod, to appear in Comm. Appl. Anal.

[9] A. Bouziani and N.-E. Benouar, Mixed problem with integral conditions for a third order parabolic equation, Kobe J. Math. 15 (1998), no. 1, 47-58.

[10] A. Bouziani and S. Mesloub, Mixed problem with a weighted integral condition for a parabolic equation with the Bessel operator, to appear in J. Appl. Math. Stochastic Anal.

[11] L. Gårding, Cauchy's Problem for Hyperbolic Equations, Lecture Notes, The University of Chicago Press, Chicago, 1957.

Abdelfatah Bouziani: Département de Mathématiques, Centre Universitaire Larbi Ben M'hidi, Oum El Bouggui, 04000, Algeria

Current address: Mathematical Division, The Abdus Salam International Centre for Theoretical Physics (ICTP), Strada Costiera 11, 34100 Trieste, Italy

E-mail address: af_bouziani@hotmail.com, bouziani@ictp.trieste.it 


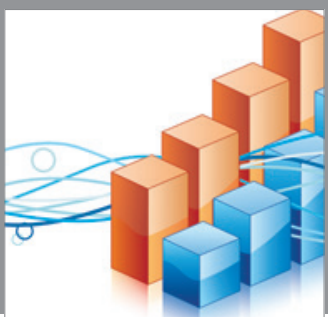

Advances in

Operations Research

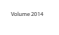

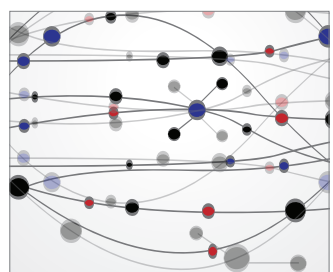

\section{The Scientific} World Journal
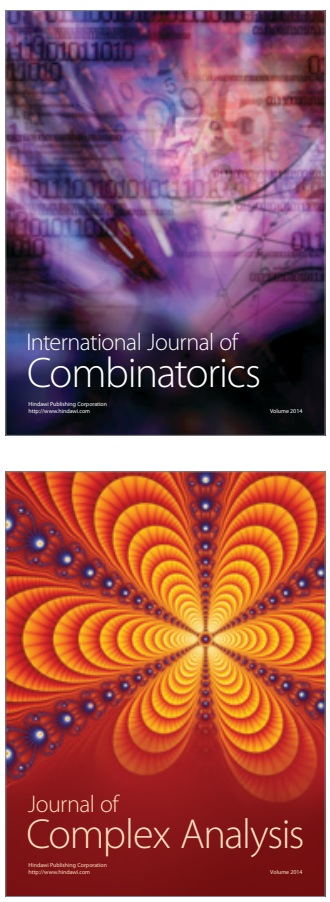

International Journal of

Mathematics and

Mathematical

Sciences
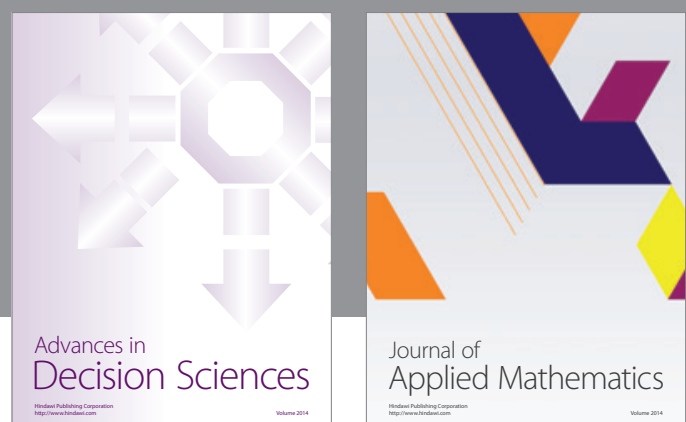

Journal of

Applied Mathematics
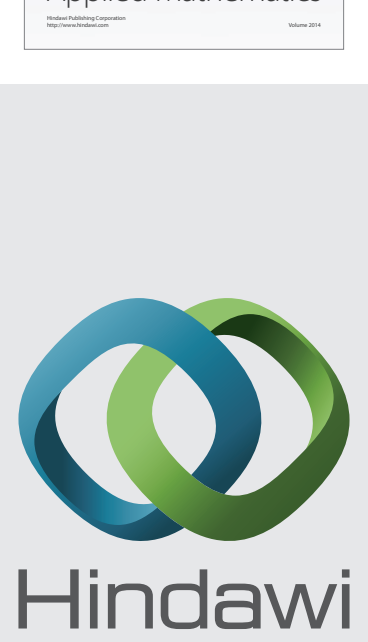

Submit your manuscripts at http://www.hindawi.com
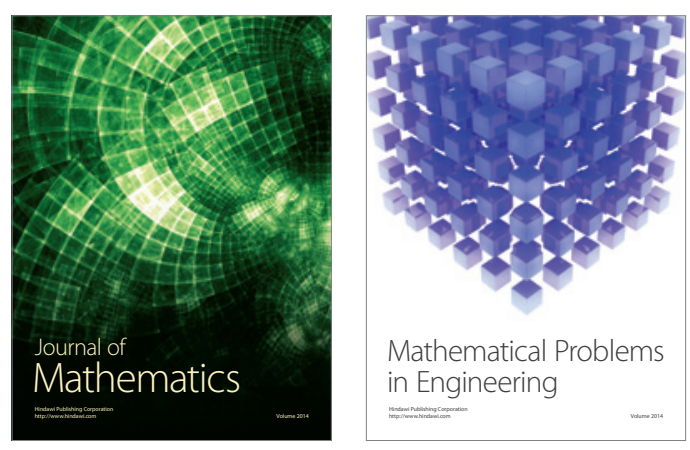

Mathematical Problems in Engineering
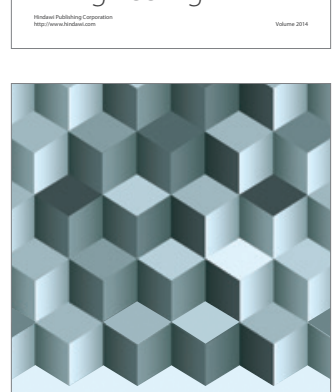

Journal of

Function Spaces
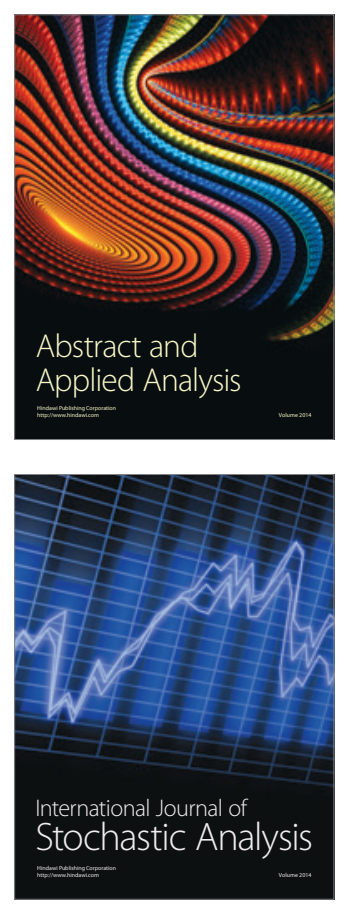

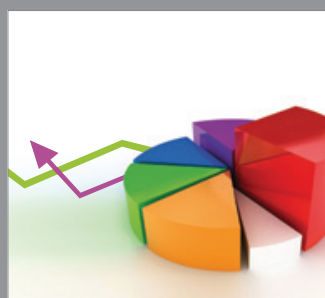

ournal of

Probability and Statistics

Promensencen
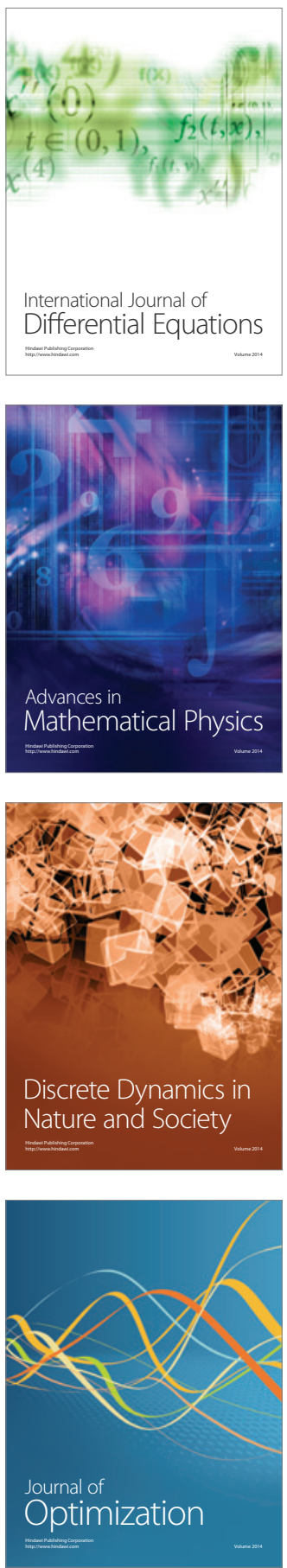